OPEN

SUBJECT AREAS:

GELS AND HYDROGELS

POLYMER CHEMISTRY

Received

28 April 2014

Accepted

20 June 2014

Published

23 July 2014

Correspondence and requests for materials should be addressed to X.T.Z. (zhangxtchina@ yahoo.com)

* These authors contributed equally to this work.

\section{Elastic, Conductive, Polymeric Hydrogels and Sponges}

\author{
Yun Lu'*, Weina He ${ }^{2 *}$, Tai Cao' ', Haitao Guo', Yongyi Zhang' ${ }^{2}$, Qingwen $\mathrm{Li}^{2}$, Ziqiang Shao', Yulin Cui ${ }^{3}$ \\ \& Xuetong Zhang ${ }^{1,2}$
}

\begin{abstract}
${ }^{1}$ School of Materials Science \& Engineering, Beijing Institute of Technology, Beijing 100081, P. R. China, ${ }^{2}$ Suzhou Institute of Nano-tech \& Nano-bionics, Chinese Academy of Sciences, Suzhou, 215123, P. R.China, ${ }^{3}$ College of Chemistry, Chemical Engineering \& Material science, Soochow University, Suzhou, 215123, P. R. China.
\end{abstract}

As a result of inherent rigidity of the conjugated macromolecular chains resulted from the delocalized $\pi$-electron system along the polymer backbone, it has been a huge challenge to make conducting polymer hydrogels elastic by far. Herein elastic and conductive polypyrrole hydrogels with only conducting polymer as the continuous phase have been simply synthesized in the indispensable conditions of 1) mixed solvent, 2) deficient oxidant, and 3) monthly secondary growth. The elastic mechanism and oxidative polymerization mechanism on the resulting PPy hydrogels have been discussed. The resulting hydrogels show some novel properties, e.g., shape memory elasticity, fast functionalization with various guest objects, and fast removal of organic infectants from aqueous solutions, all of which cannot be observed from traditional non-elastic conducting polymer counterparts. What's more, light-weight, elastic, and conductive organic sponges with excellent stress-sensing behavior have been successfully achieved via using the resulting polypyrrole hydrogels as precursors.

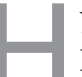
ydrogels, a classic representative of soft materials, have been widely consumed in our daily life (e.g., jellies, paints, cosmetics, and so on). Hydrogels can be made electrically conductive by embedding various conducting components (e.g., carbon, conducting polymers, metal nanomaterials) into aqueous gels mainly by physically compositing ${ }^{1-7}$ or forming co-networks ${ }^{8-12}$ for the potential uses in chemical mimicry of neural networks ${ }^{11,13-14}$, implantable electrochemical biosensors ${ }^{8,15}$, electro-stimulated drug release $e^{7,16-17}$, etc. However the performances of these conductive hydrogels would be significantly restricted by the limited electrical conductivity resulting from the leaching out of the conducting components while the hydrogel network swells or the existence of non-conductive host matrix to make electron-transferring domains ineluctably discrete ${ }^{18}$. Thus a few novel conducting hydrogels based on one component conducting polymers (e.g., conducting polymers including polyaniline (Pani), polypyrrole (PPy), polythiophene (PTh), etc.) as the continuous phase (i.e. conducting polymer hydrogels) have been developed ${ }^{19-26}$. For instance, a Pani hydrogel free of insulating polymers has been synthesized by using phytic acid as both gelator and dopant to directly form a gel network ${ }^{24}$; an amphiphilic thiophene derivative hydrogel with unusual 2D building blocks has been synthesized in one step via a combination of oxidative coupling polymerization and non-covalent crosslinking ${ }^{25}$; a PPy nanotube hydrogel with controlled morphology has been synthesized by oxidative polymerization in the presence of dye molecules as templates ${ }^{26}$. However all the obtained conducting polymer hydrogels show no elastic behaviors (See Supporting Information, Fig. SI1) which were frequently observed from some insulating polymer counterparts $^{27-32}$. On account of inherent rigidity of the conjugated macromolecular chains originated from the delocalized $\pi$-electron system along the polymer backbone ${ }^{33}$, it has been a huge challenge to make these conducting polymer hydrogels elastic by far.

Motivated by the challenges of elastic conducting polymer hydrogel formation, we sought to exploit two-step synthesis, i.e. the fast reaction and the slow reaction procedure in sequence, to prepare polypyrrole (PPy) hydrogels. The fast reaction provides the incipient network with low joint density aimed at restraining the fragility caused by high joint density. The slow reaction procedure strengthens the framework by forming homogenous polymer coatings. The assumption can be realized by several methods, among which the oxidation of pyrrole by deficient oxidant $\mathrm{Fe}\left(\mathrm{NO}_{3}\right)_{3}\left(\mathrm{Fe}^{3+}\right.$ and $\mathrm{NO}_{3}{ }^{-}$dominate the fast and slow oxidation procedures respectively), reported by this communication, is a very simple, cheap, repeatable and scalable process to synthesize elastic conductive PPy hydrogels. The resulting PPy hydrogels can be successfully converted into light-weight, elastic, conductive, organic sponges via a convenient supercritical fluid drying process. The work reported here, to the 
best of our knowledge, is the first report on the synthesis and properties of the elastic conducting polymer hydrogels (and sponges). Our work might also offer much inspiration to make more elastic conducting polymer hydrogels (as well as sponges) directly derived from Pani, PTh, etc.

\section{Results}

Syntheses of PPy hydrogel and sponge. The synthetic process for elastic PPy hydrogel is demonstrated in Figure 1A. In a brief procedure, pyrrole $(433 \mu \mathrm{L}, 6.26 \mathrm{mmol})$ was dissolved in $3.0 \mathrm{~mL}$ $\mathrm{H}_{2} \mathrm{O} /$ ethanol (v/v 1:1) mixed solvents to form colorless uniform solution. Then, a deficient amount of $\mathrm{Fe}\left(\mathrm{NO}_{3}\right)_{3} \cdot 9 \mathrm{H}_{2} \mathrm{O}(2.53 \mathrm{~g}$, $6.26 \mathrm{mmol}$ ) dissolved in $3.0 \mathrm{~mL} \mathrm{H}_{2} \mathrm{O} / \mathrm{ethanol}$ (v/v $1: 1$ ) mixed solvents were added rapidly to the pyrrole solution under vigorous agitation at $0-4{ }^{\circ} \mathrm{C}$. (Note that the sufficient amount of ferric nitrate is $7 / 3$ folds of monomer moles for the doped polymer $\left.^{34-35}\right)$. The mixed solvents allowed both of the monomers and oxidants dissolved easily. The mixture was stirred for 30 seconds before standing and formed gel in several minutes. Finally, the initial gel was grown at $25-30^{\circ} \mathrm{C}$ for 30 days (i.e. aging process). The resulting PPy hydrogel was solvent-exchanged with ethanol and supercritical $\mathrm{CO}_{2}$ in sequence to obtain the corresponding sponge.

Structural characterization. The structure of the product was measured by Fourier-transform Infrared (FT-IR) (See Fig. SI2) and Raman (See Fig. SI3) detections. FT-IR spectroscopy had confirmed the molecular structure of PPy through distinguishing vibrational band assignments. In comparison with the FT-IR and Raman spectra of PPy oxidized by equimolar amount of $\mathrm{Fe}\left(\mathrm{NO}_{3}\right)_{3}$ without and with aging process, there are no obvious changes in band positions. It can be inferred that the aging process only concerns about hydrogel network morphological changes resulting in the slow oxidization step instead of the rearrangement of molecular chains. Besides, Infra-red and Raman spectra had also confirmed that the gel framework was made of conventional PPy
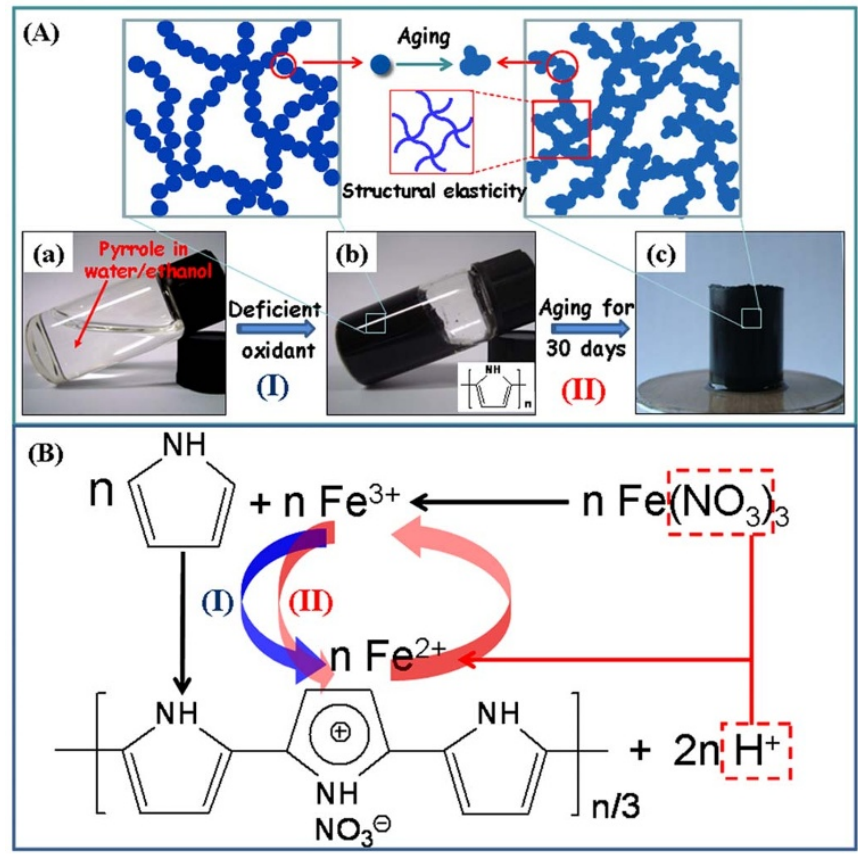

Figure 1 Schematic diagrams for the synthetic process and polymerization mechanism of the elastic polypyrrole hydrogel. (A), The synthetic process of elastic polypyrrole hydrogel. Enlarged sketch maps indicated by squares in A have shown aggregated structural change of the polypyrrole hydrogel building blocks during polymerization. (B), The polymerization mechanism of the polypyrrole hydrogel. macromolecules with quite low doping level caused by the deficient oxidation.

Super-elasticity of PPy hydrogel. To our surprise, the obtained PPy hydrogel could be compressed by $\geq 70 \%$ and recovered to its original shape in 30 seconds (Figure $2 \mathrm{a}-\mathrm{c}$ and Video S1). From the compressive stress $\sigma$ versus stain $\varepsilon$ curves for PPy hydrogels along the loading direction during loading-unloading cycles at $\varepsilon=10-70 \%$, compressive stress $\sigma$ returning to the origin after unloading for each strain $\varepsilon$ (Figure $2 \mathrm{~d}$ ). Although we found hysteresis loops in the loading-unloading cycles, indicating dissipation, that did not affect the shape recovery of the obtained hydrogles. This dissipation was negligible at $\varepsilon=10 \%$, but steadily increased with $\varepsilon$ (Figure $2 \mathrm{~d}$ ). From the comparison of the $\sigma$ vs. $\varepsilon$ curves for multi compress-release circles, the PPy hydrogel could recover their original shape with little mechanical failure under similar compression (Figure 2e). In comparison, the resulting PPy hydrogels without experiencing quite long aging progress (i.e. secondary growth) could not be elastic at all (See Fig. SI4a), just as observed from those conducting polymer hydrogels reported elsewhere ${ }^{19-26}$. These observations have shown that the secondary growth has played a key role in determining the elasticity of the resulting PPy hydrogel.

To our best knowledge, this is the first successful trial to synthesize super-elastic all conducting polymer hydrogel and sponge. The PPy$\mathrm{G}$ ( $\mathrm{G}$ stands for Graphene) foam based materials possessing similar compression-recovery properties have been published before ${ }^{36}$, but there are several considerable differences between the two materials. The biggest one is the contribution of polypyrrole to the material elasticity. In the PPy-G foam, the highly uniform deposition of PPy layers along graphene sheets increase the strength of $3 \mathrm{D}$ structure via strong $\pi-\pi$ interaction to bear a certain extra force. The source of the elasticity is still graphene sheets. However, for the PPy gels in our system, the superb elasticity comes from 3D PPy network achieved by structural design.

Origin of the super-elasticity. It has been well known that during aging of sol-gel process, two different mechanisms might affect the structure of the traditional gels: transport of materials to the neck region, and dissolution of small particles into large ones (Ostwald ripening $)^{37-38}$. There are lots of differences between aging of sol-gel process and the secondary growth of gelation procedure described here. In our case, it was observed that the monomers were oxidized into polypyrrole as soon as the oxidant was added. The insoluble polypyrrole separated from the solution to form clusters, grow into spherical particles, and connect each other to form threedimensional network through $\pi-\pi$ interaction (Figure $3 a$ ).

To explore the origin of elasticity for the obtained PPy hydrogel, real time SEM imaging and rheological recording have been done for the fresh-made PPy hydrogel during the secondary growth. After 1 day growth, many micro-protrusions began to appear on the surface of the original particles inhomogeneously (Figure $3 \mathrm{~b}$ ), which indicated that un-reacted monomers have been transferred and oxidatively coupled onto the surfaces of the PPy particles, rather than only in the neck regions between these particles. With the increase of growth time, these micro-protrusions further grew to form branches (Figure 3c). Finally these branches evolved into the ginger-like building blocks and thus jointed together randomly (Figure $3 \mathrm{~d}-\mathrm{f}$ ). It has been observed from dynamic rheology that each storage modulus (E') is always higher that the corresponding loss modulus (E'), and that both E' and E" have increased with the increase of growth time (See Fig. SI5). These observations have directly reflected further growth of PPy particles during aging process.

Thus far, we can infer that the increasing elasticity of the aged PPy hydrogel may come from the microscopic structural changes during secondary growth: (a) in comparison with the traditional synthesis of $\mathrm{PPy}$, the reaction rate is slowed down strikingly by the deficient oxidant, resulting in the reduced joint density of the incipient 

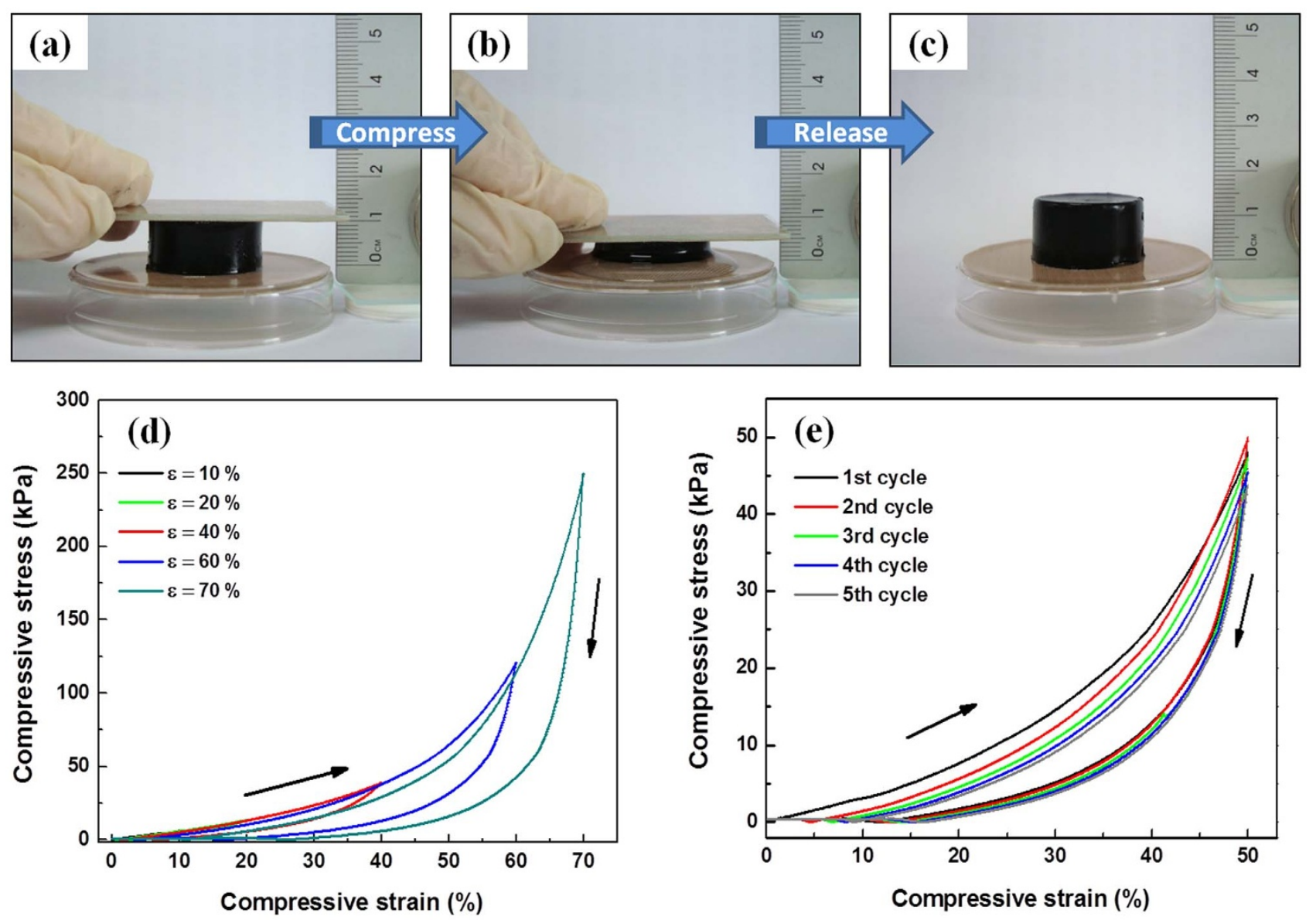

Figure $2 \mid$ Elasticity of PPy hydrogel. (a-c), Macroscopic visualization, showing that PPy hydrogel recovers their original shape after compression $\geq 70 \%$. (d) $\sigma$ versus $\varepsilon$ curves for PPy hydrogels along the loading direction during loading-unloading cycles at $\varepsilon=10-70 \%$. (e) Fatigue resistance of PPy hydrogel at $50 \%$ strain for 5 cycles.

network. As a result, the obtained PPy hydrogel exhibits less stiffness. Then, the slow reaction process in the secondary growth reinforces the initial network by coarsening the joints effectively, which avoids the structural fracture when the PPy hydrogel was compressed; (b) the asymmetrically epitaxial growth of the original particle offer many new weak contacting joints. Single-point contact mode for the building blocks of traditional PPy hydrogels under compression is replaced by muti-point contacting or even face-contacting. Taken these two possibilities into consideration, the PPy hydrogel can restrict the irreversible fracture of the hydrogel network when bearing compression.

To explore the reaction essence of the PPy secondary growth, a few comparable experiments have been designed. When sufficient ferric nitrate was used, or when deficient ferric chloride was used, to replace deficient ferric nitrate, the resulting aged PPy hydrogels showed much worse elastic performance (See Fig. SI4b, c). According to these comparisons, we can infer that the whole reaction procedure contains rapid and slow reaction stages as shown in Figure 1B. Rapid reaction stage dominates the incipient gelation of pyrrole, while the slow reaction stage is reflected in the 30 days' second-growth process. In the fast reaction stage, $\mathrm{Fe}^{3+}$ ions oxidize pyrrole to form polypyrrole at first ${ }^{39}$. With the consumption of $\mathrm{Fe}^{3+}$ ions, the reaction rate slows down. Because of the deficiency of oxidant, there are lots of monomers remaining, and the reaction gets into the slow stage ${ }^{40}$. The oxidation of pyrrole produces $\mathrm{H}^{+}$ions $\mathrm{s}^{39}$ and the generated proton can promote the oxidizability of $\mathrm{NO}_{3}{ }^{-}$. Thus, the $\mathrm{NO}_{3}{ }^{-}$ions begin to exhibit the ability to oxidize pyrrole (or oxidize $\mathrm{Fe}^{2+}$ into $\mathrm{Fe}^{3+}$, which can oxidize pyrrole further). In another word, the oxidation with $\mathrm{NO}_{3}{ }^{-}$ions dominate the slow reaction stage.
Novel properties of elastic PPy hydrogel. Excellent elasticity has endowed the resulting PPy hydrogels with some novel properties as shown in Figure 4. First of all, the PPy hydrogel maintained the compressed shape after eliminating the squeezed-out solvents $\left(\mathrm{H}_{2} \mathrm{O}\right.$ or $\left.\mathrm{CH}_{3} \mathrm{CH}_{2} \mathrm{OH}\right)$, and if fed the solvents again the hydrogel recovered their original shape (i.e., shape memory) gradually as shown in Figure $4 \mathrm{a}-\mathrm{b}$ and Video S2. Considered that solvent molecules could form hydrogen bonds with - $\mathrm{NH}$ - groups in $\mathrm{PPy}$ chains $^{41}$, these solvent molecules could act as the glue in our system. When the hydrogel was compressed, many new contacting points were generated, and new hydrogen bonds formed at the same time, which could effectively lock the compressed deformation. Providing sufficient solvent, the new formed hydrogen bonds were destroyed by the self-bonding of solvent molecules. Thus, the locked compression deformation was released.

Secondly, this shape memory elasticity could be used to realize fast functionalization of the PPy hydrogels with various nano-building blocks, e.g., $\mathrm{Fe}_{3} \mathrm{O}_{4}$ nanoparticles as shown in Figure $4 \mathrm{c}-\mathrm{e}$. When the squeezed-out solvent was removed, the PPy hydrogel remained the compressed shape. Once the $\mathrm{Fe}_{3} \mathrm{O}_{4}$ suspension was supplied, they were absorbed into the PPy hydrogel to recover the deformation. In the same time, the $\mathrm{PPy} / \mathrm{Fe}_{3} \mathrm{O}_{4}$ composite hydrogel has been prepared. The obtained $\mathrm{PPy} / \mathrm{Fe}_{3} \mathrm{O}_{4}$ composite hydrogel could be magnetic attracted by magnet (Figure $4 \mathrm{~d}$ ). From the SEM image (Figure 4e), we can see that the $\mathrm{Fe}_{3} \mathrm{O}_{4}$ nanoparticles with the diameter of around $150 \mathrm{~nm}$ were embedded in the network of PPy hydrogel.

Finally, the elastic PPy hydrogel can be also applied to extract dye molecules from the dye solutions in a very fast way. The hydrogels were placed in the tube of a syringe, and the plunger was pulled or 

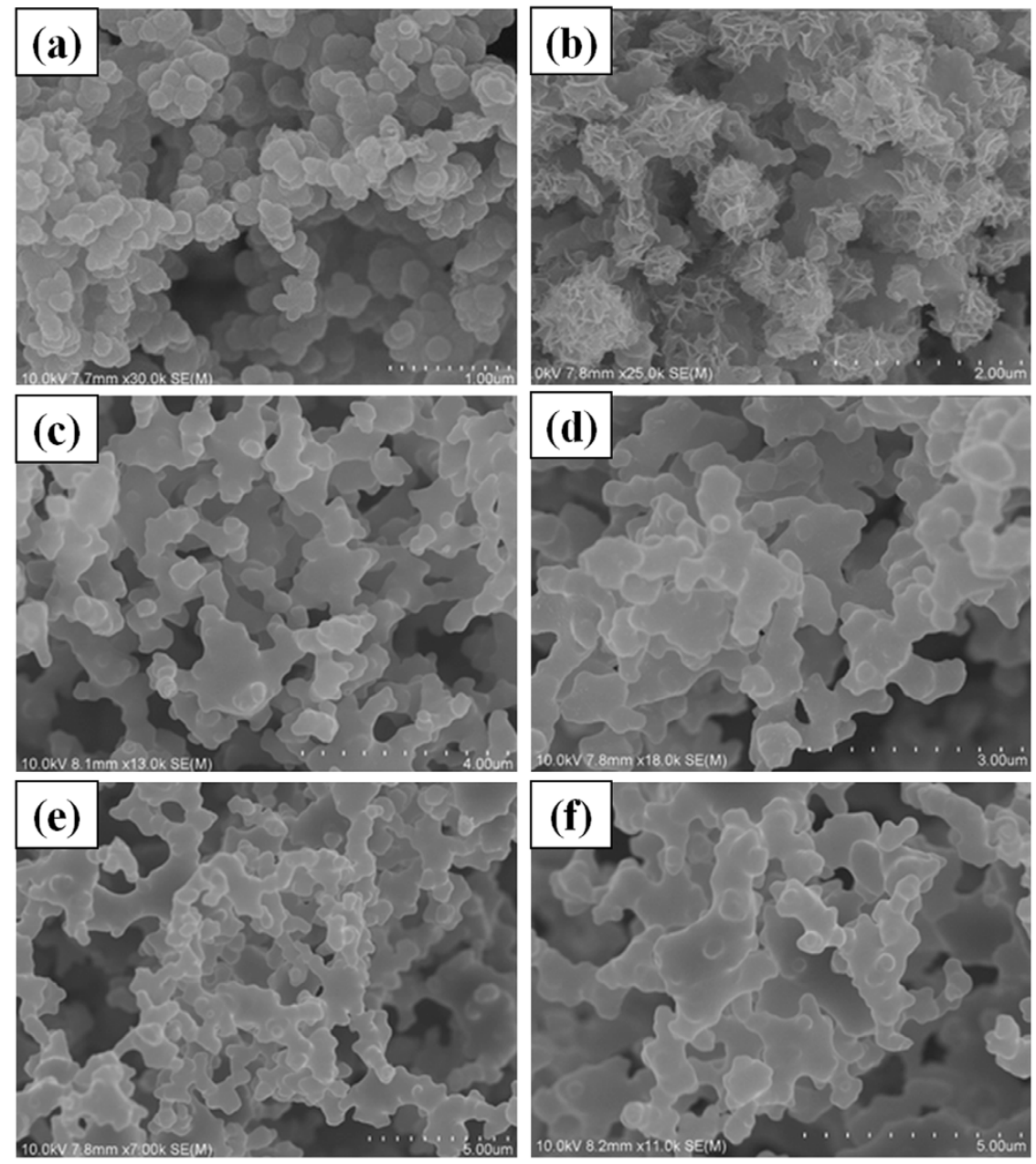

Figure 3 SEM detections of PPy hydrogels grown for different times. SEM images of PPy hydrogels grown for $0 \mathrm{~d}(\mathrm{a}), 1 \mathrm{~d}(\mathrm{~b}), 5 \mathrm{~d}(\mathrm{c}), 10 \mathrm{~d}(\mathrm{~d}), 20 \mathrm{~d}(\mathrm{e})$ and $30 \mathrm{~d}(\mathrm{f})$, respectively.

pushed allowing take in or expel the dye solutions after permeating through the PPy hydrogels. For all three kinds of dye molecules, the colorful dye solutions changed into colorless in several seconds as shown in Figure $4 \mathrm{f}-\mathrm{i}$ and Video $\mathrm{S} 3$, and the hydrogel could be used for many times. For methyl orange (MO), the dye capacity of PPy hydrogel in fast absorption mode is $389.5 \mathrm{mg} / \mathrm{g}$ with the adsorption efficiency of $99.99 \%$. Besides, the hydrogel saturated with dye molecules can be refreshed by eluting with $80^{\circ} \mathrm{C} 2 \mathrm{~mol} / \mathrm{L} \mathrm{NaOH}$ aqueous solution (See Supporting information) and the refreshed hydrogel could adsorb MO with the capacity of $196.4 \mathrm{mg} / \mathrm{g}$ and the efficiency of $99.89 \%$. The fast and effective dye absorption attributes to the high specific surface area of PPy hydrogel (ca.12 $\mathrm{m}^{2} / \mathrm{g}$ ) and strong $\pi-\pi$ interaction between dye molecules and sorbent matrix ${ }^{42-44}$. Meanwhile, the good mechanical properties of PPy hydrogels allow them to bear the dye solutions rushing through rapidly and to be used repeatedly.

Elastic, conductive and polymeric sponges. More importantly, the PPy hydrogel can be readily converted into the light-weight, elastic, conductive and organic sponges as shown in Figure 5 and Video S4. Recently, there has been growing interest in developing conductive sponges by various strategies ${ }^{45-50}$, but none of them can make allorganic sponges with desirable conductivity. However in our case, pure organic, electrically conductive (ca. $0.5 \mathrm{~S} / \mathrm{m}$ ) and light-weight $\left(70 \mathrm{mg} / \mathrm{cm}^{3}\right)$ PPy sponges (Figure $5 \mathrm{a}$ ) have been conveniently made from the resulting PPy hydrogels via simple supercritical fluid drying process. The obtained sponges have still kept the excellent elasticity after drying of the PPy hydrogels. To better explain the elasticity of the PPy sponge with the compression, SEM images of the PPy sponge under $0 \%$ and $50 \%$ compression were collected and compared (See Fig. SI6). As we can see (Fig. SI6a-b), the original sponges exhibit many macro pores with the diameter of about $10 \mu \mathrm{m}$, which will inhibit the electrical transport. However, these macro pores provide the possibility of compressed sponges to dissipate the external energy by shutting off the pores without breaking the gel framework. As the images of compressed sponges (Fig. SI6c-d) shown, the gel network became more compact and many macro pore disappear. Inevitably, many new contact points are formed and the electrical conductivity of the sponge will be raised. The gel framework of the PPy sponge did not crush by compression and still processed deformation ability. So the sponge can recover the compressive deformation and the electric conductivity will return to the original level when the compressive stress is released.

In order to explore the stress-dependent conductivity of PPy sponge during compression process, the sponge was tried to be made into stress sensors. The PPy sponge was imbedded into two parallel copper electrodes and then two copper wires were connected onto the electrodes (Figure 5b). Then, it was connected into electrical loop to detect the electrical resistance change $\left(\Delta R / R_{0}=\left(R_{0}-R\right) / R_{0}\right.$, where $\mathrm{R}_{0}$ and $\mathrm{R}$ denote the resistance without and with applied stress, respectively) when it was compressed. It can be seen (Figure 5c) that the electrical resistance decreases gradually with the compression strain, changing by $3 \%$ when it was compressed up to $80 \%$. The electrical response speed and sponge sensor stability were investigated by alternate supply of compress and release stimulations time after time. It is shown that decrease of the electrical resistance of PPy 

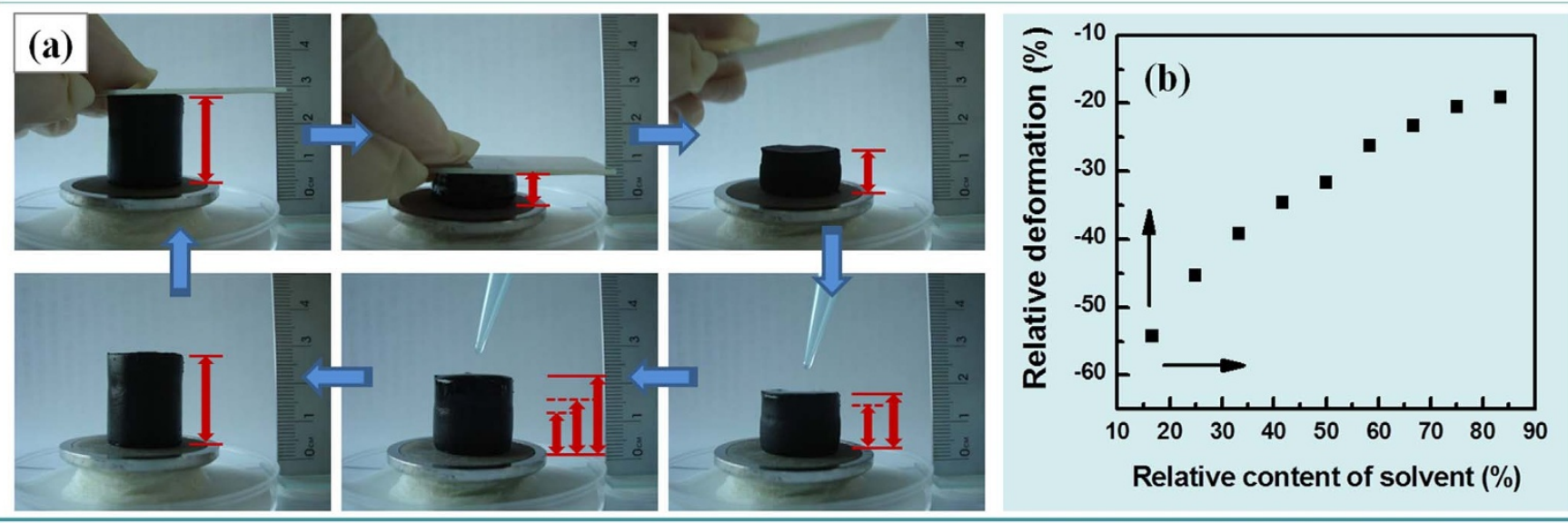

Relative content of solvent (\%)
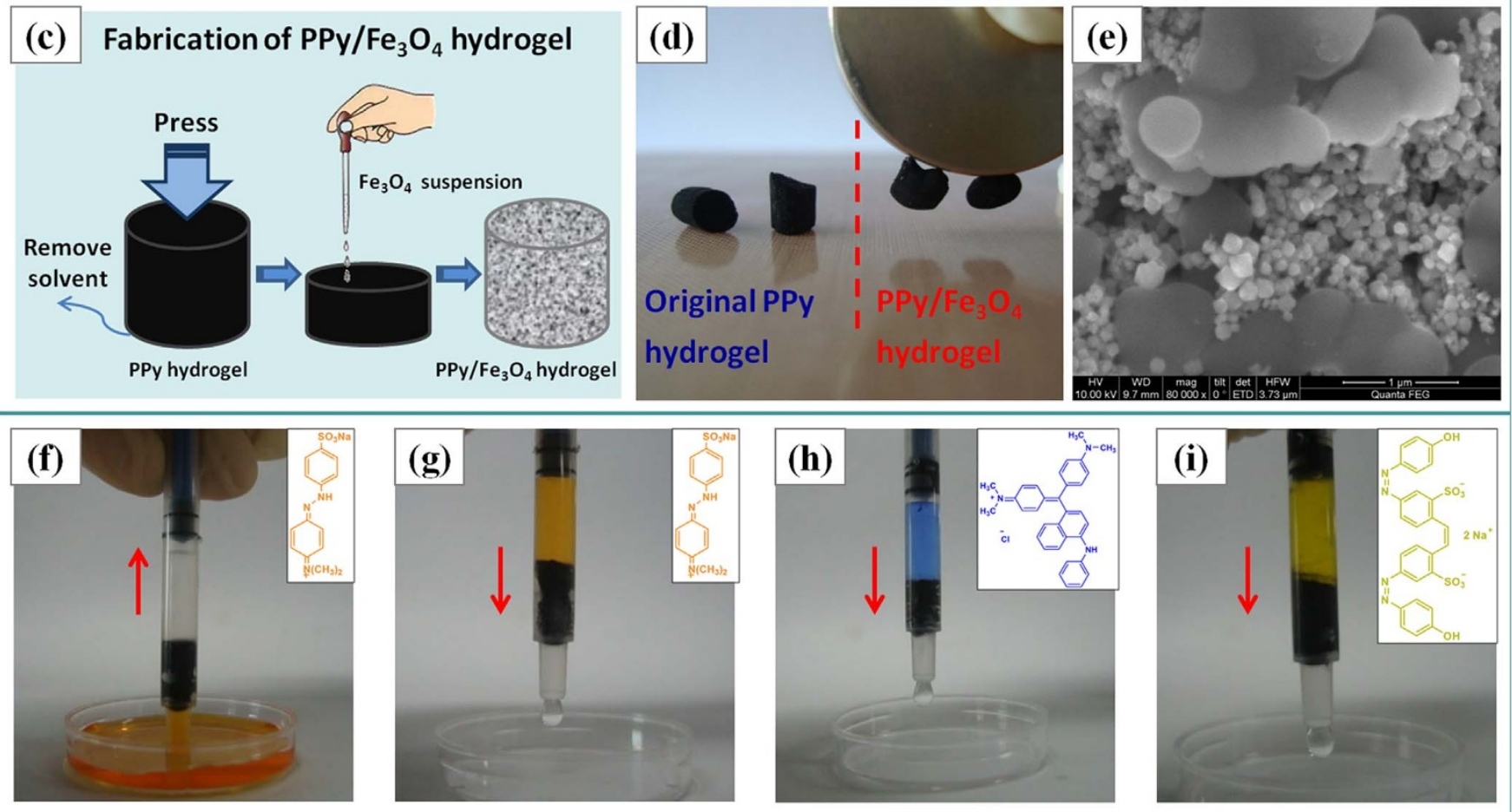

Figure $4 \mid$ Novel properties of the elastic PPy hydrogels. (a), Illustrated photos showing shape-memory elasticity of the PPy hydrogel. When PPy hydrogels were compressed and the squeezed-out solvent was removed, the PPy hydrogel kept the compressed shape. Given solvent again, PPy hydrogel recovered their shape gradually. (b), The relationship between the relative solvent content and the hydrogel deformation. (c), Schematic illustration on fast functionalizing of the PPy hydrogels with magnetic $\mathrm{Fe}_{3} \mathrm{O}_{4}$ nanoparticles. (d), Digital photo and (e), SEM image of the Fe $\mathrm{O}_{4}$ functionalized PPy hydrogels, and digital photos showing fast removal of (f) methyl orange, (g) victoria blue and (h) brilliant yellow from waste water with the elastic PPy hydrogels in syringes.

sponge resulted from the compression strain could be realized within 1.5 seconds and so was the electrical resistance recovery once sponge was released (Figure 5d). Besides, the electrical response of PPy sponge to the external stress input exhibit high stability during compression and release circles (See Fig. SI7). The decrease of electrical resistance of the compressed PPy sponge can be explicated by the more contacting points of the gel network resulting from the compression. Because of the structural elasticity of PPy sponge, the deformation recovers in seconds once the load removed and the newly-built contacts are no longer exist. Thus, the electrical resistance of the released PPy sponge returns to the original value.

\section{Discussion}

In this communication we present a very simple, cheap, repeatable and scalable process to synthesize conductive PPy hydrogels according to the following requirements: 1) pyrrole was dissolved in mixed solvents; 2) deficient oxidant was used to polymerizing the monomer and 3) long time (ca. 30 days) second-growth was occurred. The resulting PPy hydrogels have shown extraordinary elasticity with compress strain $\geq 70 \%$, and such unexpected elasticity has endowed as-made conducting polymer hydrogels with some novel properties, e.g., simple functionalization with various nanoparticles, fast removal of various dyes from waste water, shape memory effect controlled by alternately adsorbing and desorbing solvents, etc. What's more, the resulting PPy hydrogels can be conveniently converted into organic, light and conductive sponges via supercritical fluid drying process, and the resulting sponges show very excellent stress-sensing performance. All of these characteristics of the resulting PPy hydrogels and sponges render them excellent candidates for intelligent materials, environmental engineering, etc.

The smooth going of the exploration in preparing elastic PPy hydrogels and sponges opens up the possibility that the rigid con- 
(a)
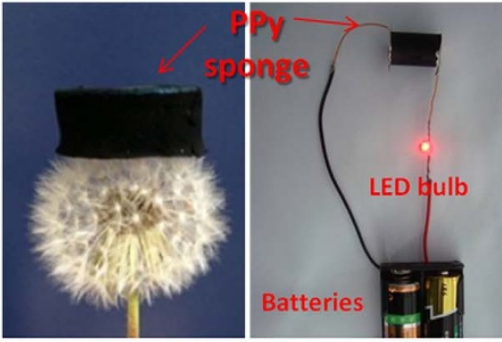

(c)

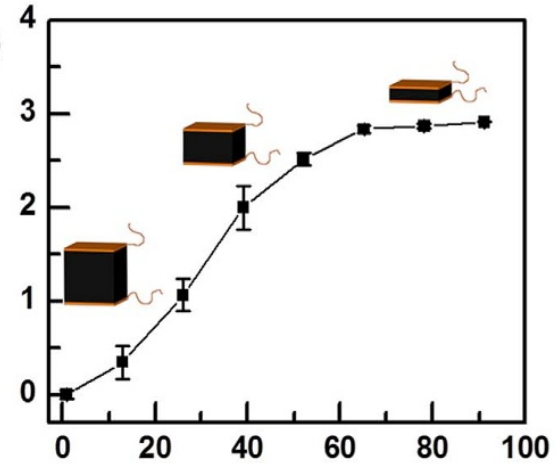

Compression strain (\%) (b)

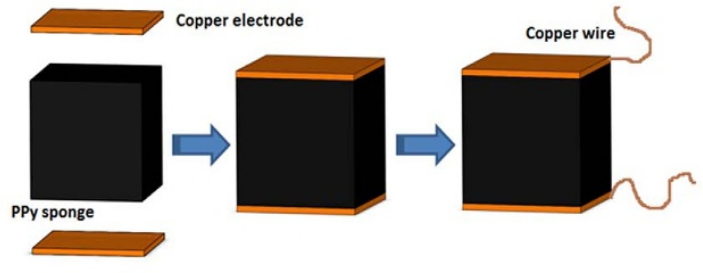

(d)

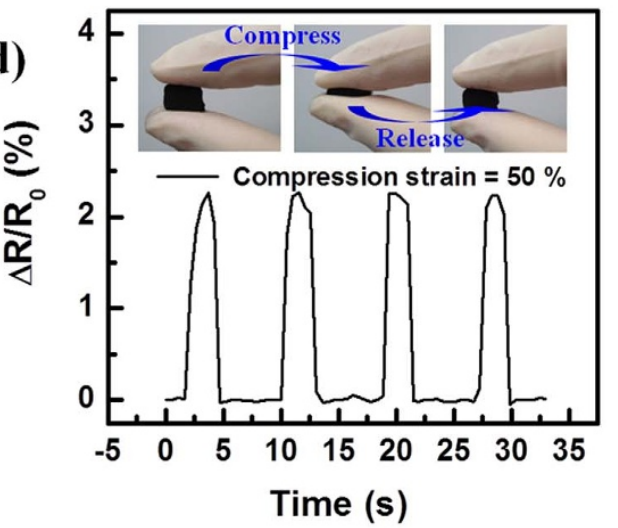

Figure 5 The light-weight, elastic, conductive and organic sponges made from the resulting PPy hydrogels via supercritical fluid drying process. (a), Digital photos of PPy sponges located on a dandelion and connected to the LED bulb. (b), Schematic illustration of how to prepare a stress sensor used by PPy sponge, copper sheets and metal wires. (c), Change of electrical resistance of the PPy sponge sensor with the compression ratio (the errors bars represent one standard deviation) and (d), change of electrical resistance of the PPy sponge sensor with the compression and release circles at $\varepsilon=50 \%$. Inset in $\mathrm{d}$ : macroscopic visualization, showing that PPy sponge recovers their original shape after compression.

ducting polymers can be processed into elastic porous materials. Stepwise growth procedure in synthesizing conducting polymer hydrogels helps us improve 3D network and strength the framework independently. Furthermore, the combination of different kinds of oxidants has been proved to be an effective way to realize stepwise oxidation. Thus, our work might offer much inspiration to make more elastic conducting polymer hydrogels (as well as sponges) directly derived from Pani, PTh, etc.

\section{Methods}

Chemicals. The pyrrole was distilled under reduced pressure before use. All the other chemicals were of analysis grade and used as received. The synthesis procedures on various conducting polymer hydrogels are described as below. If it was not mentioned specially, the obtained hydrogels were purified via solvent exchange process with a large amount of deionized water to remove unreacted monomer and other impurites.

Synthesis of Polypyrrole hydrogel. Oxidized by deficient $\mathrm{Fe}\left(\mathrm{NO}_{3}\right)_{3}$ : The elastic PPy hydrogel was synthesized by mixing pyrrole monomer and equimolar amount of $\mathrm{Fe}\left(\mathrm{NO}_{3}\right)_{3}$ in $\mathrm{H}_{2} \mathrm{O}$ /ethanol ( $\left.\mathrm{v} / \mathrm{v} 1: 1\right)$ mixed solution at $0^{\circ} \mathrm{C}$ and aging at $25-30^{\circ} \mathrm{C}$ for 30 days. In order to illustrate the contribution of aging procedure, the fresh made hydrogel was compressed and released to probe the hydrogel elasticity.

Oxidized by sufficient $\mathrm{Fe}\left(\mathrm{NO}_{3}\right)_{3}$ :In order to compare the elasticity of PPy hydrogel synthesized by fast oxidation, the pyrrole was mixed with sufficient $\mathrm{Fe}\left(\mathrm{NO}_{3}\right)_{3}\left(\mathrm{nFe}^{3+}\right.$ : npyrrole $=2.3: 1)$ in $\mathrm{H}_{2} \mathrm{O}$ /ethanol $(\mathrm{v} / \mathrm{v} 1: 1)$ mixed solution at $0^{\circ} \mathrm{C}$, and then placed at room temperature for $2 \mathrm{~h}$ to form PPy hydrogel.

Oxidized by deficient $\mathrm{FeCl}_{3}$ :In order to emphasize the dominant effect of $\mathrm{NO}_{3}{ }^{-}$in the slow oxidation step described in our work, $\mathrm{PPy}$ hydrogel was synthesized by equimolar amount of $\mathrm{FeCl}_{3}$ in $\mathrm{H}_{2} \mathrm{O}$ /ethanol (v/v $1: 1$ ) mixed solution at $0^{\circ} \mathrm{C}$, and then placed at room temperature for 30 days to form PPy hydrogel.

Synthesis of various conducting polymer hydrogels for comparison. Pani hydrogel: The chemical oxidative polymerization of aniline is carried out according to the mothod reported by Bao Zhenan et al. ${ }^{24}$ In a typical synthesis, $0.921 \mathrm{~mL}(1 \mathrm{mmol})$ phytic acid (50\%, wt/wt in water) and $0.458 \mathrm{~mL}$ aniline were dissolved in $2 \mathrm{~mL} \mathrm{DI}$ water at first. Then $1 \mathrm{~mL}$ oxitant solution containing $0.286 \mathrm{~g}(1.25 \mathrm{mmol})$ ammonium persulfate aqueous solution was added to that mixture at $4{ }^{\circ} \mathrm{C}$ with violent agitation. The reaction mixture was placed at room temperature for 24 hours to obtain Pani hydrogels.
PTh derivative hydrogel: PTh derivative hydrogel was prepared according to the procedure reported in our previous study ${ }^{25}$ by oxidizing sodium 4-(2,3-dihydrothieno[3,4-b] [1,4] dioxin-2-yl) methoxybutane-1 -sulfonate (EDOT-S) monomer with iron(III) chloride $\left(\mathrm{FeCl}_{3}\right)$ in DI water. In a typical procedure, the prepared EDOT-S monomer ( $40 \mathrm{mg}, 0.12 \mathrm{mmol}$ ) dissolved in $0.1 \mathrm{~mL}$ deionized water, and $\mathrm{FeCl}_{3}(392.8 \mathrm{mg}, 2.42 \mathrm{mmol})$ in $0.7 \mathrm{~mL}$ DI water were mixed and stirred for $\sim 30 \mathrm{~s}$ to ensure complete mixing. Subsequently the reaction proceeded without agitation for $24 \mathrm{~h}$ at room temperature. Thus, the PEDOT-S hydrogel was been prepared.

Polypyrrole Nanotube hydrogel: polypyrrole hydrogel synthesized via template of $\mathrm{Fe}\left(\mathrm{NO}_{3}\right)_{3} \cdot \mathrm{MO}(\mathrm{MO}=$ sodium 4 -[4-(dimethylamino) phenyldiazo] phenylsulfonate $\left.\left(\mathrm{CH}_{3}\right)_{2} \mathrm{NC}_{6} \mathrm{H}_{4} \mathrm{~N}\right] \mathrm{NC}_{6} \mathrm{H}_{4} \mathrm{SO}_{3} \mathrm{Na}$, Methyl orange) as described in literature ${ }^{26} .0 .303 \mathrm{~g}$ of $\mathrm{Fe}\left(\mathrm{NO}_{3}\right)_{3} \cdot 9 \mathrm{H}_{2} \mathrm{O}(0.75 \mathrm{mmol})$ was first added into $10 \mathrm{~mL} \mathrm{MO}$ aqueous solution with the concentration of $5 \mathrm{mmol} / \mathrm{L}$. Then $0.5 \mathrm{mmol}$ of pyrrole was added. The resulting system was stirred for $2 \mathrm{~min}$ and then placed at room temperature for two hous to form PPy hydrogel.

Characterization. The structure of the product was measured by Fourier-transform Infrared (FT-IR) and Raman detections. The PPy hydrogel samples were mashed, washed, filtered, and dried under vacuum before measurement. FT-IR spectra were recorded on a Magna-IR 750 spectrometer. The powdered PPy hydrogel samples were milled with potassium bromide $(\mathrm{KBr})$ to form fine powders. The powders were then compressed into a thin pellet for analysis. Raman spectra were recorded on a LavRAM Aramis spectrometer with $50 \mathrm{~mW}$ He-Ne laser operating at $632.8 \mathrm{~nm}$ with a CCD detector. The powdered PPy hydrogel samples were placed on slides for detection. The morphologies of the obtained PPy hydrogels oxidized by equimolar $\mathrm{Fe}\left(\mathrm{NO}_{3}\right)_{3}$ were examined by SEM (Hitachi S-4800) with the acceleration voltage of $10 \mathrm{KV}$. PPy hydrogel samples aging for different time periods were purified in a large amount of deionized water and dried at room temperature under atmospheric pressure. The dried samples for SEM experiments were set on conducting stages and observed with gold coatings. The aging procedure of PPy hydrogel was tracked by dynamic rheological measurements operated on Physica MCR 301 apparatus at $25^{\circ} \mathrm{C}$. The storage modulus $\left(\mathrm{G}^{\prime}\right)$ and loss modulus $\left(\mathrm{G}^{\prime \prime}\right)$ of the hydrogel samples were measured at the angular frequency ranging from 0.1 to $100 \mathrm{rad} / \mathrm{s}$. The compressive stress-strain measurements were performed on the elastic PPy hydrogel by using a BESTE KJ-1065 tensile testing machine at a crosshead speed of $5 \mathrm{~mm} / \mathrm{min}$. The conductivity of obtained hydrogels and sponges were measured by testing the electric current of series circuit which contained the tested samples under $1 \mathrm{~V}$ input voltage.

Performance tests. The shape-memory property of the elastic PPy hydrogel was demonstrated by setting the hydrogel sample onto the stacked filter papers to absorb the squeezed solvent rapidly when the hydrogels were compressed. Subsequently, the solvent were added dropwise from the top of the squeezed hydrogel. The relationship 
between the amount of the solvent added and deformation recovery of PPy hydrogel was recorded and the corresponding diagram was made. In order to illustrate that the PPy hydrogels can be easily functionalized by absorbing nanoparticle suspensions, $\mathrm{Fe}_{3} \mathrm{O}_{4}$ magnetic nanoparticles (MNPs) were produced according to the literature at first ${ }^{51}$. Briefly, $1.39 \mathrm{~g}(0.005 \mathrm{~mol})$ of ferrous sulfate $\left(\mathrm{FeSO}_{4}\right)$ and $1.24 \mathrm{~g}(0.005 \mathrm{~mol})$ of sodium thiosulfate $\left(\mathrm{Na}_{2} \mathrm{~S}_{2} \mathrm{O}_{3}\right)$ was dissolved with $14 \mathrm{~mL}$ distilled water in a Teflonlined stainless autoclave, followed by dropwise addition of $10 \mathrm{~mL} \mathrm{NaOH}(1.0 \mathrm{~mol} / \mathrm{L})$ solution to produce a black colloid medium. The autoclave was maintained at $140^{\circ} \mathrm{C}$ for 12 hours, and then allowed to cool to room temperature. The obtained $\mathrm{Fe}_{3} \mathrm{O}_{4}$ MNPs, extracted by using a magnet, were washed by a large amount of deionized water. The purified $\mathrm{Fe}_{3} \mathrm{O}_{4}$ MNPs were dissolved in deionized water to form suspension, which was added to the compressed PPy hydrogel at last. Methyl orange, Victoria blue and Bright yellow were selected to illustrate the fast absorption of dye molecules with the PPy hydrogel. Three kinds of dye aqueous solutions changed into colorless after passing through purified PPy hydrogel which had been put into the tube of a syringe. Quantitative data of dye removal were collected using a V-660 UVvis spectrometer (Jasco International Co., Tokyo, Japan). The absorbance spectra of filtered solvent were determined by UV-vis spectroscopy detection, and dye concentrations were analyzed by comparing absorption intensity at its absorbance maximum with the standard curves. Methyl orange (MO) was selected as the representative of the dye molecules.

The amount of adsorbed MO was calculated by Eq. (1):

$$
\mathrm{Q}_{\mathrm{t}}=\frac{\left(\mathrm{C}_{0}-\mathrm{C}_{\mathrm{t}}\right) \mathrm{V}}{\mathrm{M}}
$$

where $\mathrm{Q}_{t}$ is the dye capacity in the polypyrrole hydrogel placed in the tube of a syringe $(\mathrm{mg} / \mathrm{g}), \mathrm{C}_{0}$ is the initial dye concentration in aqueous solution $(\mathrm{mg} / \mathrm{mL}), \mathrm{C}_{\mathrm{t}}$ is the dye concentration in the filtered solution $(\mathrm{mg} / \mathrm{mL}), \mathrm{V}$ is the total volume of dye solution permeated through hydrogel in which the dye can't be detected after filtered $(\mathrm{mL})$, and $\mathrm{M}$ is the mass of sorbent used (g).

And the absorption efficiency was calculated by Eq. (2):

$$
\mathrm{E}(\%)=\frac{\mathrm{C}_{0}-\mathrm{C}_{\mathrm{e}}}{\mathrm{C}_{0}} \times 100 \%
$$

The PPy sponge was made by supercritical $\mathrm{CO}_{2}$ drying of the as-made PPy hydrogel. Two parallel copper electrodes were pasted onto the top and bottom surface of PPy sponge by brushing silver conductive epoxy adhesive, and then two copper wires were connected onto the electrodes. The electrical resistance of PPy sponge under compression was measured by testing the electric current of series circuit which contained the tested samples under $1 \mathrm{~V}$ input voltage. In order to trace the electrical resistance changes during compression, the PPy sponge was compressed from $\varepsilon=0 \%$ to $90 \%$ slowly in 30 seconds. The PPy sponge suffered instant alternate compression and release to investigate the resistance response rate to external stimuli.

1. Annabi, N. et al. 25th Anniversary Article: Rational Design and Applications of Hydrogels in Regenerative Medicine. Adv. Mater. 26, 85-124 (2014).

2. Justin, G. \& Guiseppi-Elie, A. Characterization of Electroconductive Blends of Poly(HEMA-co-PEGMA-co-HMMA-co-SPMA) and Poly(Py-co-PyBA). Biomacromolecules 10, 2539-2549 (2009).

3. Bai, H., Sheng, K., Zhang, P., Li, C. \& Shi, G. Graphene oxide/conducting polymer composite hydrogels. J. Mater. Chem. 21, 18653-18658 (2011).

4. Molina, M. A., Rivarola, C. R., Miras, M. C., Lescano, D. \& Barbero, C. A. Nanocomposite synthesis by absorption of nanoparticles into macroporous hydrogels. Building a chemomechanical actuator driven by electromagnetic radiation. Nanotechnol. 22, 245504 (2011).

5. Xia, Y. Y. \& Zhu, H. L. Polyaniline nanofiber-reinforced conducting hydrogel with unique pH-sensitivity. Soft Matter 7, 9388-9393 (2011).

6. Chikar, J. A. et al. The use of a dual PEDOT and RGD-functionalized alginate hydrogel coating to provide sustained drug delivery and improved cochlear implant function. Biomaterials 33, 1982-1990 (2012).

7. Ge, J., Neofytou, E., Cahill, T. J., Beygui, R. E. \& Zare, R. N. Drug Release from Electric-Field-Responsive Nanoparticles. ACS Nano 6, 227-233 (2012).

8. Mano, N., Yoo, J. E., Tarver, J., Loo, Y. L. \& Heller, A. An electron-conducting cross-linked polyaniline-based redox hydrogel, formed in one step at pH 7.2, wires glucose oxidase. J. Am. Chem. Soc. 129, 7006-7007 (2007).

9. Sun, X. M. et al. Two-step synthesis of superabsorbent conducting hydrogel based on poly(acrylamide-pyrrole) with interpenetrating network structure. Mater. Res. Innovations 15, 70-74 (2011).

10. Jin, L. et al. Dual signal glucose reporter based on inverse opal conducting hydrogel films. Soft Matter 8, 4911-4917 (2012).

11. Lu, Y. et al. Poly(3,4-ethylenedioxythiophene)/poly(styrenesulfonate)-poly(vinyl alcohol)/poly(acrylic acid) interpenetrating polymer networks for improving optrode-neural tissue interface in optogenetics. Biomaterials 33, 378-394 (2012).

12. Xiao, Y., He, L. \& Che, J. An effective approach for the fabrication of reinforced composite hydrogel engineered with SWNTs, polypyrrole and PEGDA hydrogel. J. Mater. Chem. 22, 8076-8082 (2012).

13. Nyberg, T., Inganäs, O. \& Jerregård, H. Polymer Hydrogel Microelectrodes for Neural Communication. Biomed. Microdevices 4, 43-52 (2002).
14. Kim, D. H., Abidian, M. \& Martin, D. C. Conducting polymers grown in hydrogel scaffolds coated on neural prosthetic devices. J. Biomed. Mater. Res. Part A 71A, 577-585 (2004)

15. Brahim, S., Narinesingh, D. \& Guiseppi-Elie, A. Polypyrrole-hydrogel composites for the construction of clinically important biosensors. Biosens. Bioelectron. 17, $53-59(2002)$

16. Murdan, S. Electro-responsive drug delivery from hydrogels. J. Control. Release 92, 1-17 (2003)

17. Lira, L. M. \& de Torresi, S. I. C. Conducting polymer-hydrogel composites for electrochemical release devices: Synthesis and characterization of semiinterpenetrating polyaniline-polyacrylamide networks. Electrochem. Commun. 7, 717-723 (2005).

18. Lin, J., Tang, Q., Wu, J. \& Li, Q. A multifunctional hydrogel with highconductivity, $\mathrm{pH}-$ responsive, and release properties from polyacrylate/ polyptrrole. J. Appl. Polym. Sci. 2010, 116, 1376-1383 (2010).

19. Mawad, D. et al. A Single Component Conducting Polymer Hydrogel as a Scaffold for Tissue Engineering. Adv. Funct. Mater. 22, 2692-2699 (2012).

20. Du, R. \& Zhang, X.-T. Alkoxysulfonate-Functionalized Poly (3,4ethylenedioxythiophene) Hydrogels. Acta Physico-Chimica Sinica 28, 2305-2314 (2012).

21. Chen, L., Kim, Nishino, M., Gong, J. P. \& Osada, Y. Environmental Responses of Polythiophene Hydrogels. Macromolecules 33, 1232-1236 (2000).

22. Marcasuzaa, P., Reynaud, S., Ehrenfeld, F., Khoukh, A. \& Desbrieres, J. Chitosangraft-Polyaniline-Based Hydrogels: Elaboration and Properties. Biomacromolecules 11, 1684-1691 (2010).

23. Dai, T., Jiang, X., Hua, S., Wang, X. \& Lu, Y. Facile fabrication of conducting polymer hydrogels via supramolecular self-assembly. Chem. Commun. 4279-4281 (2008).

24. Pan, L. et al. Hierarchical nanostructured conducting polymer hydrogel with high electrochemical activity. PNAS 109, 9287-9292 (2012).

25. Du, R., Xu, Y., Luo, Y., Zhang, X. \& Zhang, J. Synthesis of conducting polymer hydrogels with $2 \mathrm{D}$ building blocks and their potential-dependent gel-sol transitions. Chem. Commun. 47, 6287-6289 (2011).

26. Wei, D. et al. Controlled growth of polypyrrole hydrogels. Soft Matter $\mathbf{9}$, 2832-2836 (2013).

27. Abdurrahmanoglu, S., Can, V. \& Okay, O. Equilibrium swelling behavior and elastic properties of polymer-clay nanocomposite hydrogels. J. Appl. Polym. Sci. 109, 3714-3724 (2008)

28. Gaharwar, A. K., Dammu, S. A., Canter, J. M., Wu, C. J. \& Schmidt, G. Highly Extensible, Tough, and Elastomeric Nanocomposite Hydrogels from Poly(ethylene glycol) and Hydroxyapatite Nanoparticles. Biomacromolecules 12, 1641-1650 (2011).

29. Sun, J. Y. et al. Highly stretchable and tough hydrogels. Nature 489, 133-136 (2012).

30. Wen, Q., Basu, A., Janmey, P. A. \& Yodh, A. G. Non-affine deformations in polymer hydrogels. Soft Matter 8, 8039-8049 (2012).

31. Singh, N. S. Effect of surfactants on polyvinyl alcohol-borax hydrogel: Rheology and thermal aspects. Indian J. Chem. Sect A-Inorg. Bio-Inorg. Phys. Theor. Anal. Chem. 52, 879-883 (2013).

32. Xia, L.-W. et al. Nano-structured smart hydrogels with rapid response and high elasticity. Nat. Commun. 4, 2226 (2013).

33. Wijsboom, Y. H. et al. Controlling Rigidity and Planarity in Conjugated Polymers: Poly(3,4-ethylenedithioselenophene). Angew. Chem. Int. Ed. 48, 5443-5447 (2009).

34. Machida, S., Miyata, S. \& Techagumpuch, A. Chemical synthesis of highly electrically conductive polypyrrole. Synthetic Mel. 31, 311-318 (1989).

35. Pecher, J. \& Mecking, S. Nanoparticles of Conjugated Polymers. Chem. Rev. 110, 6260-6279 (2010).

36. Zhao, Y. et al. Highly Compression-Tolerant Supercapacitor Based on Polypyrrole-mediated Graphene Foam Electrodes. Adv. Mater. 25, 591-595 (2013).

37. Brinker, C. J. \& Scherer, G. W. Sol Gel Science: The Physics and Chemistry of SolGel Processing (Academic Press, New York, 1990).

38. Woignier, T. \& Phalippou, J. Mechanical strength of silica aerogels. J. Non-Cryst. Solids 100, 404-408 (1988).

39. Myers, R. E. Chemical oxidative polymerization as a synthetic route to electrically conducting polypyrroles. J. Electron. Mater. 15, 61-69 (1986).

40. Planche, M. F., Thiéblemont, J. C., Mazars, N. \& Bidan, G. Kinetic study of pyrrole polymerization with iron (III) chloride in water. J. Appl. Polym. Sci. 52, 1867-1877 (1994).

41. Schiavon, G., Zotti, G., Comisso, N., Berlin, A. \& Pagani, G. Ion Exchange in the Electrochemical Switching of Polypyrroles in Acetonitrile by the Electrochemical Quartz Crystal Microbalance. Electrolyte Incorporation by Hydrogen Bonding of Anions to Pyrrole. J. Phys. Chem. 98, 4861-4864 (1994).

42. Håkansson, E., Lin, T., Wang, H. \& Kaynak, A. The effects of dye dopants on the conductivity and optical absorption properties of polypyrrole. Synthetic Mel. 156, 1194-1202 (2006).

43. Jia, H. et al. Molecular Dynamics Simulation of Interactions on Graphene/ Polypyrrole Nanocomposites Interface. Integr. Ferroelectr. 145, 130-139 (2013).

44. Qian, T., Yu, C. F., Wu, S. S. \& Shen, J. A facilely prepared polypyrrole-reduced graphene oxide composite with a crumpled surface for high performance supercapacitor electrodes. J. Mater. Chem. A 1, 6539-6542 (2013). 
45. Luo, X. \& Cui, X. T. Sponge-like nanostructured conducting polymers for electrically controlled drug release. Electrochem. Commun. 11, 1956-1959 (2009).

46. Zhang, X., Chang, D., Liu, J. \& Luo, Y. Conducting polymer aerogels from supercritical $\mathrm{CO}_{2}$ drying PEDOT-PSS hydrogels. J. Mater. Chem. 20, 5080-5085 (2010).

47. Zhang, X., Li, C. \& Luo, Y. Aligned/Unaligned Conducting Polymer Cryogels with Three-Dimensional Macroporous Architectures from Ice-Segregation-Induced Self-Assembly of PEDOT-PSS. Langmuir 27, 1915-1923 (2011).

48. Xu, Y., Sui, Z., Xu, B., Duan, H. \& Zhang, X. Emulsion template synthesis of all conducting polymer aerogels with superb adsorption capacity and enhanced electrochemical capacitance. J. Mater. Chem. 22, 8579-8584 (2012).

49. Antonio, J. L. S., Lira, L. M., Goncales, V. R. \& Cordoba de Torresi, S. I. Fully conducting hydro-sponges with electro-swelling properties tuned by synthetic parameters. Electrochim. Acta 101, 216-224 (2013).

50. Temmer, R., Kiefer, R., Aabloo, A. \& Tamm, T. Direct chemical synthesis of pristine polypyrrole hydrogels and their derived aerogels for high power density energy storage applications. J. Mater. Chem. A 1, 15216-15219 (2013).

51. Fan, R., Chen, X. H., Gui, Z., Liu, L. \& Chen, Z. Y. A new simple hydrothermal preparation of nanocrystalline magnetite $\mathrm{Fe}_{3} \mathrm{O}_{4}$. Mater. Res. Bull. 36, 497-502 (2001).

\section{Acknowledgments}

This work was financially supported by the National Natural Science Foundation of China (21373024), the Innovation Program of the Beijing Institute of Technology and the 100

Talents Program of the Chinese Academy of Sciences.

\section{Author contributions}

Y.L., W.H., T.C. and X.Z. designed the experiment. Y.L., W.H. and T.C. performed synthesis experiments and characterization. W.H. and Y.C. performed super-elasticity analysis of PPy hydrogel. W.H. designed the experiment and performed the novel properties analysis of PPy hydrogel and sponges. W. H. and X.Z. wrote the paper. Y.L., W.H., T.C., H.G., Y.Z., Q.L., Z.S. and X.Z. contributed to analysis the experiment data.

\section{Additional information}

Supplementary information accompanies this paper at http://www.nature.com/ scientificreports

Competing financial interests: The authors declare no competing financial interests. How to cite this article: Lu, Y. et al. Elastic, Conductive, Polymeric Hydrogels and Sponges. Sci. Rep. 4, 5792; DOI:10.1038/srep05792 (2014).

This work is licensed under a Creative Commons Attribution-NonCommercialShareAlike 4.0 International License. The images or other third party material in this article are included in the article's Creative Commons license, unless indicated otherwise in the credit line; if the material is not included under the Creative Commons license, users will need to obtain permission from the license holder in order to reproduce the material. To view a copy of this license, visit http:// creativecommons.org/licenses/by-nc-sa/4.0/ 